\title{
Consumo alimentar de população adulta residente em área rural da cidade de I batiba (ES, Brasil)
}

\author{
Consumption to feed of resident adult population \\ in rural area of the city of Ibatiba (ES, Brazil)
}

Edilaine Oliveira Carvalho ${ }^{1}$

Emersom Ferreira da Rocha ${ }^{1}$

${ }^{1}$ Centro Universitário de Barra M ansa, Campus Barra M ansa. Rua Vereador Pinho de Carvalho 267, Centro. 27330-550 Barra Mansa RJ lanalengg@yahoo.com.br
Abstract It is a transverse study where a questionnaire of alimentary frequency was applied (QAF) in 150 adults resident of the rural area of the city of I batiba (ES, Brazil). QAF classified the alimentary consumption as: habitual ( $\geq 4$ times in the week), not habitual ( $<4$ times in the week) and rarely ( 1 time a month), with objective of correlating the alimentary consumption with the chronic-degenerative diseases. The results evidenced a habitual consumption of rice, breads, stalk, bean, cow milk, animal fat, margarine, sugar and coffee, and a non habitual consumption of cake, potato, cookies, manioc, sweet potato, chayote, carrot, beet, pumpkin, juice of fruits, banana, orange, guava, mango and tangerine. It can be concluded that the feeding habit presented by the studied population it can come to increase in a medium or long period the prevalence and occurrences of chronic-degenerative diseases as hypertension, diabetes, obesity and coronary diseases. The alimentary consumption of this population needs concern, because when compared with the national patterns, it is observed some inadequacies, and it is known that this picture comes to every day causing damages the public health. Key words Feeding consumption, Chronic-degenerative non-transmissible diseases, Balanced feeding, Rural population
Resumo Trata-se de um estudo transversal no qual foi aplicado um questionário de frequência alimentar (QFCA) em 150 adultos residentes em área rural da cidadedel batiba (ES). 0 QFCA classificou o consumo alimentar como: habitual ( $\geq 4$ vezes na semana), não habitual ( $<4$ vezes na se mana) e raramente (uma vez ao mês), com o fim de correlacionar o consumo alimentar encontrado com o fato de risco ou não para a ocorrência de doenças crônicas não transmissíveis diretamente relacionadas à alimentação que afetam a população brasileira hoje. Os resultados evidenciaram consumo habitual de arroz, pães, folhosos, feijão, leite de vaca, gordura animal, margarina, açúcar ecafé, e um consumo não habitual debolo, batata, biscoitos, aipim, batata-doce, chuchu, cenoura, beterraba, abóbora, suco defrutas, banana, laranja, goiaba, manga e tangerina. Conclui-se que 0 hábito alimentar da população estudada pode vir a incrementar a médio ou longo prazo a prevalência e/ ou ocorrência de doenças crônicas não transmissíveis, sobretudo as relacionadas à alimentação, como hi pertensão, diabetes, obesidadeedoenças coronárias. 0 consumo alimentar dessa população requer preocupação, pois se comparado aos padrõesnacionais, observam-sealgumasinadequações, esabe se que este quadro vem a cada dia ocasionando danos à saúde pública.

Palavras-chave Consumo alimentar, D oenças crônicas não transmissíveis, Alimentação equilibrada, População rural 
Introdução

Em países em desenvolvimento como o Brasil é cediço que a estrutura agrária está caracterizada pela grande concentração de terra em mãos de alguns poucos proprietários, em que pese a permanência de grupos populacionais de assalariados rurais ou não vivendo em precárias condições de vida. No caso do Brasil, com o nível existente de miséria e pobreza da população, há a necessidade de o Estado intervir no sentido de melhorar as condições gerais da sociedade, através de programas assistenciais que incluam oferta de boa instrução e alimentação.

0 estudo do consumo alimentar de uma população é capaz de gerar uma série de informações que, de certa forma, refletem a sua organização social, uma vez que a alimentação constitui necessidade prioritária em qual quer sociedade².

0 perfil alimentar da população está fortemente associado a aspectos culturais, nutricionais, socioeconômicos e demográficos, tornando necessário um melhor entendimento desses aspectos eseus mecanismos no enten dimento das mudanças de comportamento alimentar e suas consequências ${ }^{3}$. A avaliação dos hábitos alimentares de populações apresenta-se como tarefa importante a cada dia, tendo em vista os estudos já realizados que relacionam a alimentação tanto com a prevenção quanto com o tratamento de diversas patologias4.

Os efeitos benéficos de uma dieta equilibrada e da prática de exercícios físicos na prevenção de doenças crônicas são amplamente divulgados e reconhecidos na literatura 5 . A alimentação adequada édireito fundamental do ser humano, inerente à dignidade da pessoa humana e indispensável à realização dos direitos consagrados na Constituição Federal, devendo o poder público adotar as políticas e ações que se façam necessárias para promover e garantir a segurança alimentar enutricional da população ${ }^{6}$. N este sentido, este artigo refere-se aos resultados de uma pesquisa que busca mapear o consumo alimentar de uma população rural específica e relacioná-lo com os riscos de ocorrência de doenças crônicas não transmissíveis, às quais o fator dietético está fortemente associado.

O Brasil, ao lado da maioria dos países da América Latina, da África e da Ásia, se depara com as novas epidemias de obesidade, diabetes, osteoporose, doenças cardíacas e câncer do pulmão, do cólon edo reto, da mama, da próstata e outras. Esse peso multiplicado das doenças, sujeito a se tornar ainda pior à medida que a popu- lação brasileira aumenta e envelhece, não pode ser abordado apenas com tratamentos médicos e cirúrgicos, apesar de estes serem de importância vital. M esmo em países de maior renda, o custo do tratamento das doenças crônicas não transmissíveis constitui um enorme encargo social eeconômico? ${ }^{7}$.

\section{Metodologia}

Trata-se de um estudo transversal, no qual foi aplicado um questionário de frequência alimen$\operatorname{tar}$ (QFCA) em uma amostra populacional selecionada aleatoriamente de 150 indivíduos adultos na faixa etária entre 20 e 59 anos, residentes em zona rural na cidade de Ibatiba, estado do Espírito Santo.

Deacordo com o censo demográfico de2000, a população do município de l batibaé de 19.210 indivíduos, sendo 10.596 depopulação urbanae 8.614 depopulação rural; em relação ao sexo feminino, a população éde 9.443; eao sexo masculino, de 9.763 indivíduos. Em relação à composição, étnica temse: brancos, negros e pardos, que estão distribuídos de acordo com os seguintes percentuais: $56,36 \% ; 3,11 \%$ e $40,53 \%$, respectivamente 8 .

Em relação aos aspectos econômicos, a exemplo de outros municípios capixabas, a economia local possui como principal atividade a produção de café. A região é caracterizada pela predominância de minifúndios rurais, monocultores em sua maioria. A comercialização do caféé realizada por intermediários sediados no município, e o leiteé direcionado para as cooperativas e algumas empresas da região. Os produtos agrícolas são vendidos no comércio local, em municípios vizinhos, produzidos para subsistência, e o excedente édirecionado para o comércio local.

A amostra foi estabelecida através de cálculo estatístico no qual foi considerado o valor de $8 \%$ para margem de erro ${ }^{9}$. A metodologia mais conveniente, tendo em vista a proposta do trabalho, baseou-se na seguinte fórmula: $n=N * n \% N+n^{\circ}$, em que $\mathrm{N}$ é o número da população de análise que corresponde a 4.241 indivíduos ${ }^{10}$. Neste número, estão englobados todos os adultos de ambos os sexos de 20 a 59 anos de idade residentes nesta área rural.

E para o cálculo do $n^{\circ}$ que representa a primeira estimativa da população, utilizou-se a fórmula: $1 / \mathrm{E}^{\circ 2}$, em que $\mathrm{E}^{\circ}$ é 0 erro tolerável de $8 \%$ que foi usado neste trabal ho. De posse do tamanho da amostra ideal, também foi analisado o procedimento mais adequado para se fazer essa 
coleta de dados. Há métodos como amostragem casual simples, amostragem estratificada, amostragem sistemática, amostragem por conglome rado; optou-se então pela praticidade da amostragem casual simples, em que qualquer pessoa desta população teve chance de ser avaliada?.

O QFCA se dedicou à evidenciação da frequência alimentar, sendo os alimentos classificados em grupos, os quais permitiram a análise do objeto de estudo deste trabalho. Tais grupos de alimentos foram divididos em: (1) pães, tubérculos, cereais e raízes; (2) hortaliças, verduras e legumes; (3) óleos e gorduras; (4) frutas; (5) leguminosas; (6) carnes e ovos; (7) produtos lácteos; (8) doces. Foi definido ainda um subgrupo que correspondia aos alimentos diversos (café, produtos integrais, refrigerantes, salgados). Tal metodologia, questionário de frequência alimentar qualitativo (QFCA), foi privilegiada, pois foram considerados o seu baixo custo e sua fácil aplicabilidade na análise do hábito alimentar de indivíduos ou grupos populacionais.

A fácil aplicabilidade do QFCA provém do fato deo entrevistado ter queresponder somente se consome ou não determinado alimento, e em caso positivo, se ele consome menos que quatro vezes na semana, sendo a frequência neste caso classificada como não habitual; maior ou igual a quatro vezes na semana, classificada como habitual; e uma vez no mês classificada como raramente consumida ${ }^{11}$.

Foi investigada também a frequência das refeições diárias (desjejum, colação, almoço, lanche da tarde, jantar eceia) para verificar o ritmo alimentar desses indivíduos por intermédio da combinação de duas ou mais refeições diárias, considerando como adequada a realização de pelo menos três refeições diárias ${ }^{11}$.

Para aplicação do QFCA, o pesquisador foi até a casa dos entrevistados. Eles tomaram ciência da pesquisa através do Termo de Consentimento LivreeEsclarecido, aprovado pelo Comitê de Ética do Centro Universitário de Barra $M$ ansa. Cada entrevistado foi arguído sobre o consumo dos alimentos do seu próprio plantio, como também sobre o consumo dos animais que criavam. Isto foi feito com o objetivo de verificar de que forma o consumo desses alimentos se expressava em seus hábitos alimentares.

\section{Resultados}

Em relação à variável sexo, 68\% dos entrevistados correspondiam ao sexo feminino e $32 \%$ ao sexo masculino. Conforme os resultados, verificou-se que dentre os alimentos produzidos por esta população se destacam: arroz, feijão, milho, abóbora, laranja, batata-doce, aipim, café, canade-açúcar, banana, tomate, hortaliças, chuchu, manga, goiaba, abacate e limão. Além destes alimentos, esta população mantém criação de animais para o consumo, como: bovino, suíno, aves epeixes.

Dosalimentos consumidos habitualmente $(\geq$ quatro vezes/semana), destacam-se arroz, pães, folhosos, feijão, leite de vaca, gordura animal, margarina, açúcar, farinha de mandioca e café, e dos alimentos raramente consumidos (uma vez ao mês), destacam-se soja, lentilha, iogurte, rapadura, cana-de-açúcar, alimentos integrais, salgados, balas e caramelos, jabuticaba (Quadro 1).

Sobre os alimentos consumidos não habitualmente (<quatro vezes/semana) nos diferentes grupos de alimentos, pôde-se observar maior percentual em relação aos alimentos consumidos habitualmente dos seguintes itens: bolo, batata, biscoito recheado e sal gado, ai pim, batatadoce, chuchu, cenoura, beterraba, abóbora, suco de frutas, banana, Iaranja, goiaba, manga, tangerina, limão, abacate, carnesuína, carnebovina, peixes, ovos, aves, queijo, óleo vegetal, melado e refrigerantes (Quadro1).

Sobre a frequência com que os entrevistados realizam as suas refeições diárias, os resultados evidenciam que $52 \%$ deles realizam três refeições/ dia, $24 \%$ duas refeições/dia, $18 \%$ quatro refeições/dia, $6 \%$ cinco refeições/dia.

No grupo deprodutoslácteos, observa-seque $60 \%$ das mulheres consomem leite, porém somente 30\% consomem este alimento habitualmente. Em relação aos demais produtos lácteos como queijos eiogurtes, eles são consumidospelas mulheres na ordem de $50 \%$ e $42 \%$, respectivamente, entretanto correspondem a $30 \%$ e $14 \%$ do consumo não habitual.

\section{Discussão}

Os problemas decorrentes do consumo inadequado de alimentos já são conhecidos há muito tempo, expondo a população a graves e flagrantes danos à saúde, principalmente se consideramos os diferenciais regionais existentes no país. Estudos observacionais têm evidenciado estreita relação entre características qualitativas da dieta e ocorrência de enfermidades crônicas não transmissíveis como as doen ças cardiovasculares, diabetes mellitus, câncer e obesidade. Todas essas 
Quadro 1. Distribuição da frequência de consumo alimentar segundo grupos de alimentos. População adulta de área rural, I batiba (ES), 2006.

\begin{tabular}{|c|c|c|c|c|c|}
\hline Grupo de alimentos & $\underset{\%}{\operatorname{Sim}}$ & $\begin{array}{l}\text { Não } \\
\%\end{array}$ & $\begin{array}{c}\geq 4 \text { vezes/semana } \\
\%\end{array}$ & $\begin{array}{c}<4 \text { vezes/semana } \\
\%\end{array}$ & $\begin{array}{c}1 \text { vez/mês } \\
\%\end{array}$ \\
\hline \multicolumn{6}{|l|}{ Pães, cereais, raízes e tubérculos } \\
\hline Arroz & 100 & - & 88 & 12 & - \\
\hline Pães & 92 & 8 & 54 & 36 & 2 \\
\hline Farinha de mandioca & 80 & 20 & 48 & 24 & 8 \\
\hline Bolo & 88 & 12 & 12 & 64 & 12 \\
\hline Batata & 96 & 4 & 26 & 64 & 6 \\
\hline Biscoito rech/sal & 68 & 32 & 6 & 48 & 14 \\
\hline Aipim & 82 & 18 & 8 & 48 & 26 \\
\hline Batata-doce & 86 & 14 & 6 & 56 & 24 \\
\hline \multicolumn{6}{|l|}{ Hortaliças, verduras e legumes } \\
\hline Folhosos & 86 & 14 & 52 & 34 & - \\
\hline Chuchu & 74 & 26 & 26 & 40 & 8 \\
\hline Cenoura & 86 & 14 & 12 & 50 & 24 \\
\hline Beterraba & 74 & 26 & 6 & 42 & 26 \\
\hline Abóbora & 90 & 10 & 20 & 50 & 20 \\
\hline \multicolumn{6}{|l|}{ Frutas } \\
\hline Suco de frutas & 86 & 14 & 16 & 58 & 12 \\
\hline Banana & 84 & 16 & 16 & 66 & 2 \\
\hline Laranja & 86 & 14 & 22 & 52 & 12 \\
\hline Goiaba & 76 & 24 & 10 & 38 & 28 \\
\hline M anga & 82 & 18 & 12 & 46 & 24 \\
\hline Jabuticaba & 72 & 28 & 8 & 30 & 34 \\
\hline Tangerina & 82 & 18 & 8 & 40 & 34 \\
\hline Limão & 84 & 16 & 10 & 54 & 20 \\
\hline Abacate & 76 & 24 & 12 & 40 & 24 \\
\hline \multicolumn{6}{|l|}{ Leguminosas } \\
\hline Feijão & 94 & 6 & 84 & 10 & - \\
\hline Soja & 14 & 86 & - & 2 & 12 \\
\hline Lentilha & 16 & 84 & - & 4 & 12 \\
\hline \multicolumn{6}{|l|}{ Carnes e ovos } \\
\hline Carne suína & 74 & 26 & 26 & 32 & 16 \\
\hline Carne bovina & 90 & 10 & 26 & 58 & 6 \\
\hline Peixes & 84 & 16 & - & 48 & 36 \\
\hline Ovos & 94 & 6 & 8 & 72 & 14 \\
\hline Aves & 94 & 6 & 4 & 82 & 8 \\
\hline \multicolumn{6}{|l|}{ Produtos lácteos } \\
\hline Leite de vaca & 76 & 24 & 38 & 36 & 2 \\
\hline Queijo & 66 & 34 & 10 & 42 & 14 \\
\hline logurte & 48 & 52 & - & 18 & 30 \\
\hline \multicolumn{6}{|l|}{ Óleos e gorduras } \\
\hline Gordura animal & 82 & 18 & 74 & 4 & 4 \\
\hline Óleo vegetal & 70 & 30 & 18 & 48 & 4 \\
\hline M argarina & 94 & 6 & 62 & 32 & - \\
\hline \multicolumn{6}{|l|}{ Doces } \\
\hline Açúcar & 96 & 4 & 80 & 16 & - \\
\hline Melado & 64 & 36 & 8 & 32 & 24 \\
\hline Rapadura & 74 & 26 & 8 & 22 & 44 \\
\hline Balas/caramelos & 62 & 38 & - & 16 & 52 \\
\hline Caldo de cana & 68 & 32 & - & 16 & 52 \\
\hline \multicolumn{6}{|l|}{ Diversos } \\
\hline Café & 82 & 38 & 64 & 12 & 6 \\
\hline Alimentos integrais & 28 & 72 & - & 4 & 24 \\
\hline Salgados & 60 & 40 & 8 & 22 & 30 \\
\hline Refrigerantes & 66 & 34 & 10 & 34 & 22 \\
\hline
\end{tabular}


patologias constituem, hoje, um problema prioritário de Saúde Pública?.

Aspectos singulares da transição nutricional ocorrida no século passado são encontrados em cada país e região do mundo, mas elementos comuns convergem para uma dieta rica em gorduras (particularmente de origem animal), açúcar e alimentos refinados, e reduzida em carboidratos complexos e fibras, frequentemente denominada "dieta ocidental"12.

Os achados deste estudo destacam um consumo habitual de gordura animal eaçúcar etambém um consumo não habitual de frutas que são fontes ricas em fibras, embora os entrevistados tenham fácil acesso a este grupo de alimento. Isto é, embora as frutas sejam um alimento regionalmentecultivado, elas não são consumidas diariamente. Isto pode ser explicado por váriosfatores; entre eles, podemos citar o período de safra e entressafra característico deste grupo de alimento, como também se avalia que provavelmenteo cultivo deste alimento destina-se muito mais à comercialização e muito pouco ao consumo.

Estudos realizados em nível nacional, como foi o caso das Pesquisas de Orçamento Familiar (POFs) e o Estudo N acional de Despesa Familiar (Endef), ocorridos nas décadas de 60, 70, 80 e nos anos de 2002/03, mostraram em seus resultados mudanças importantes no padrão alimentar do brasileiro.

Essa mudança caracteriza-se pela redução do consumo de cereais e derivados, feijão, raízes e tubérculos, observada principalmente na passagem da década de 70 para a de 80 , pelo aumento contínuo no consumo de ovos, leite e derivados, substituição da banha, toucinho e manteiga por óleos vegetais e margarina, aumento no consumo de carnes, principalmente a partir da segunda metade da década de $70^{7,13}$. No entanto, em nosso estudo, realizado numa população rural, observa-se que a gordura animal é consumida habitualmente por mais de $70 \%$ desta população, ou seja: os entrevistados consomem diariamenteesse tipo degordura, em discordância, de certo modo, com os achados em âmbito nacional. Conclui-se que neste aspecto existe ainda um traço dietético de permanência, provavelmente por se tratar de área rural, contrapondo-se aos estudos supracitados que privilegiaram a zona urbana.

Em relação aos produtos lácteos, observa-se um consumo habitual de leite de vaca, considerando o consumo de homens e mulheres, porém os derivados do leite, como o queijo e o iogurte, são alimentos não habitualmente consumidos. As carnes e os ovos são alimentos consumidos menos que quatro vezes na semana, considerando a frequência para cada tipo de carne.

No que se diz respeito à associação entre hábito alimentar e risco de ocorrência de doenças crônicas não transmissíveis, há evidências deque os fatores genéticos são capazes de modular a resposta do organismo às variações dos fatores ambientais, como dieta e atividade física; e a ingestão energética aumentada eo gasto energético diminuído têm sido apontados como os principais fatores causais da obesidade. Além do valor calórico total, é importante a composição da dieta, sendo fator de risco para a obesidade uma dieta rica em carboidrato simples e lipídeos ${ }^{14}$.

De acordo com os dados do Datasus em relação às doenças crônicas não transmissíveis, a população de I batiba apresenta o seguinte perfil de mortalidade (considerando o coeficiente do número de óbitos no ano de 2005 por 100.000 habitantes): diabetes, 27,9; doenças cérebro-cardiovasculares, 51,2; infarto agudo do miocárdio, 41,9; Portanto, são dados de notória importância, que podem ser perniciosamenteincrementados dianteda manutenção dehábitos alimentares ede vida que configurem risco direto à saúde $\mathrm{e}^{15}$.

De acordo com o panorama epidemiológico no Brasil, as deficiências nutricionaise as infecções ainda são desafios fundamentais da Saúde Pública no Brasil. Ao mesmo tempo, o perfil epidemiológico adquiriu maior complexidade, tendo os padrões de doenças mudado radicalmente. As doenças crônicas não transmissíveis vêm assumindo importante magnitude, estando associadas às causas mais comuns de morte registradas atualmente $^{7}$. N este sentido, os resultados encontrados neste trabalho evidenciam que o hábito alimentar encontrado (consumo habitual de carboidratos simples e complexos, sobretudo os açúcares, arroz, pães efarinhas; gorduras, especialmente as de origem animal e margarinas) concorre para o incremento na ocorrência da obesidade, que pode gerar transtornos metabólicos importantes como aqueles que geralmente a acompanham: hipertensão, cardiopatias, diabetes mellitus, entre outros.

Sobre a presença de cálcio na dieta, os avanços dos estudos sobrea interação entrenutrientes reforçam a dificuldade dese obter sucesso na prevenção da osteoporose sem uma intervenção multifaceada e precoce. 0 consumo de dietas que atendam às recomendações de cálcio e nas quais esteestejabiodisponível deveser incentivado como uma das estratégias da prevenção de osteoporose primária, tornando-senecessário investir em educação nutricional para crianças, jovens eadultos ${ }^{16}$.

Entretanto, ao atentarmos para este aspecto, 
em nosso estudo foi observado que as mulheres não têm o hábito deconsumir leite eseus derivados, o que pode representar um fator de risco para as doenças ligadas ao metabolismo do cálcio como a osteoporose, hoje importante problema para a saúde da mulher, especialmente no âmbito da coletividade.

\section{Conclusão}

De certo modo, a partir dos resultados breve mente comparados com os padrões nacionais, podemos observar um consumo habitual de arroz, pães, folhosos, feijão, leite de vaca, gordura animal, margarina, açúcar, cafée farinha demandioca - de maneira que o consumo habitual desses alimentos se deve ao fato de eles serem obje tos de plantio, provavelmente pouco valorizado no mercado. Então o hábito de consumi-los se associa ao fácil acesso, com isso eles terminam por exceder no consumo.

Do ponto de vista da transição nutricional, conclui-se que a população rural de l batiba pode ser relativamente caracterizada por uma "dieta ocidental" em quesetem um consumo significativo de gorduras principalmente de origem animal, como também de carboidratos simples. E sabeseque estetipo de dieta está intimamenterelacionado com as doenças crônicas não transmissíveis, podendo-se afirmar que populações querealizam estetipo de dieta podem a longo ou médio prazo adquirir doenças como diabetes mellitus, obesidade, hipertensão e doenças coronarianas.

Em relação à frequência das refeições diárias, conclui-sequea maioria dos en trevistados realiza uma alimentação adequada, segundo o número de refeições por dia, pois consomem cerca detrês refeições/dia. Conclui-setambém que esta população tem uma ingestão reduzida de produtos lácteos, o que é preocupante no caso das mulheres, pois são as mais vulneráveis à osteoporose.

Entre os fatores mais importantes associados às doenças crônicas não transmissíveis, podemos destacar que em termos de hábitos alimentares rurais existe a necessidade de se aprofundarem os estudos - porque parece preocupante a situação alimentar da população rural, ainda queapresenteparticularidades quelheconferem alguma proteção, como é o caso do fator dietético como o consumo de fibras - prevenindo-se assim as possíveis doenças crônicas não transmissíveis.

\section{Colaboradores}

EO Carvalho realizou o levantamento bibliográfico, a coleta de dados, análise e interpretação dos resultados; EF Rocha contribuiu no levantamento bibliográfico, na interpretação dos resultados e na supervisão da pesquisa. 


\section{Referências}

1. Silva MS. Avaliação de adequação nutricional dos alimentos consumidos em um Centro Integrado de Educação Pública (Ciep). Cad Saude Publica 1995; 4(11):559-552

2. Romani SM, Amigo H. Perfil alimentar e posse de terra na área rural do estado de Pernambuco, Nordeste do Brasil. Rev Saude Publica 1986; 5(20):376369.

3. Bonomo E, Caiaffa WT, César SC, Lopes ACS, Costa MFL. Consumo alimentar da população adulta segundo perfil socioeconômico e demográfico: Projeto Bambuí. Cad Saude Publica 2003; 5(19):69-57.

4. Cervato AM, Vieira VL. Consumo alimentar: como avaliar a qualidade. Rev Nutrição em Pauta 2003; 2:16-12.

5. N unes FB, Lanzillotti HS, Portella ES, Soares EA Estudo comparativo do consumo alimentar de universitárias. Rev Nutrição Brasil 2005; 2(4):86-80.

6. Brasil. Lei $n^{\circ} 11.346$, de 15 de setembro de 2006 . Dispõe sobre Criação do Sistema Nacional de Segurança Alimentar e Nutricional-SISAN com vistas a assegurar o direito humano à alimentação adequada e dá outras providências. Diário Oficial da União 2006; 15 set.

7. Brasil. M inistério da Saúde. Guia alimentar para a população brasileira. Brasília: M inistério da Saúde; 2005.

8. Instituto de Pesquisas e Estudos Sociais (IPES). Estrutura etária da população por situações de domicílio e sexo. [site da Internet] [acessado 2006 jun 15]. Disponível em: www.ipes.es.gov.br/perfil/pdf/ municipios/30/demografia/tab01.pdf

9. Martins GA, DonaireD. Princípios de estatística. São Paulo: Atlas; 1979. p. 3-255.

10. Instituto de Pesquisas e Estudos Sociais (IPES).. Perfil municipal: demografia, população residente. [site da Internet] [acessado 2006 jun 15]. Disponível em: www.ipes.es.gov.br/perfil/pdf/municipios/ 30/demografia/tab01.pdf

11. Santos SJ, Costa MCO, Nascimento Sobrinho CL, Silva M CM, Souza KEP, M elo BO. Perfil antropométrico e consumo alimentar de adolescentes de Teixeira de Freitas - Bahia, Brasil. Rev de Nutrição 2005; 5(18):623-632.
12. M onteiro CA, M ondini L, Souza ALM, Popkin BM. Da desnutrição para a obesidade: a transição nutricional no Brasil. In: Monteiro CA. Velhos e novos males da saúde no Brasil: a evolução do país e de suas doenças. São Paulo: Hucitec; 2006. p. 248-255.

13. Mondini $L, M$ onteiro $C A$. M udanças no padrão de alimentação. In. Monteiro CA. Velhos e novos males da saúde no Brasil: a evolução do país e de suas doenças. São Paulo: Hucitec; 2006; p. 80-89.

14. Balaban G, Silva GAP. Efeito protetor do aleitamento materno contra a obesidade infantil. Jor da Pediatria 2004; 1(80):7-16.

15. Brasil. Ministério da Saúde. Caderno de Informações de Saúde: informações gerais município de Ibatiba. [site da Internet] [acessado 2008 mar 20]. Disponível em: http//w3.datasus.gov.Br/datasus/datasus.php

16. Lanzillotti HS, Lanzillotti RS, Trotte APR, Dias AS, Bornand S, Costa EAM M. Osteoporose em mulheres na pós-menopausa: cálcio dietético e outros fatores de risco. Rev de Nutrição 2003; 2(16):181-193.

Artigo apresentado em 18/12/2007

A provado em 11/04/2008

Versão final apresentada em 11/05/2008 